\title{
Rotaciones y niveles de razonamiento, según el modelo de Van Hiele: resultados de una experiencia
}

\author{
MERCEDES MARITZA SARRÍN SUÁREZ \\ Escuela Superior de Guerra Aérea - Perú \\ Recibido el 28-02-17; primera evaluación el 29-06-18; segunda evaluación el 12-07-18; \\ tercera evaluación el 19-11-18; aceptado el 08-02-19
}

\section{RESUMEN}

Este artículo presenta los resultados de una investigación cualitativa de diseño etnográfico, realizada con ocho (8) estudiantes de $5^{\circ}$ grado de educación secundaria de la Institución Educativa Fernando Belaúnde Terry de Ate. El objetivo de la investigación es conocer el desarrollo del pensamiento geométrico en el tema rotaciones, según el modelo Van Hiele. La recolección de datos se realizó mediante un seguimiento al desarrollo de las actividades programadas en el módulo de aprendizaje de rotaciones diseñado según el modelo Van Hiele, la prueba formativa de respuestas abiertas y la entrevista mixta. Los resultados muestran que los estudiantes, en su mayoría, exhibieron características del nivel 2 (análisis) con un buen camino hacia la adquisición de características del nivel 3 (clasificación).

Palabras clave: pensamiento geométrico, módulo de aprendizaje de rotaciones, modelo de Van Hiele.

\section{Rotations and Levels of Reasoning, according to the Van Hiele model: Results of an Experience}

\section{Abstract}

This article presents the results of a qualitative research of ethnographic design, carried out with eight (8) students of fifth grade of the Fernando Belaúnde Terry secondary school of Ate, the objective of the investigation was to know the

\footnotetext{
* Licenciada y bachiller en Educación en la Universidad Nacional Federico Villarreal, segunda especialidad en Tecnología Educativa y maestría en Medición, Evaluación y Acreditación de la Calidad de la Educación en la Universidad Nacional Mayor de San Marcos. Asesora docente de la Escuela Superior de Guerra Aérea del Perú y en Enseñanza en Instituciones Educativas Públicas especialidad Matemática. Correo: mercedessarrin@gmail.com
} 
development of the geometric thought of rotations in the students, according to the Van Hiele model. The data collection was done through a follow up to the development of the activities programmed in the rotation-learning module designed according to the Van Hiele model, the formative test of open answers and the mixed interview. The results show that students mostly exhibited characteristics of level 2 (analysis) with a good path towards the acquisition of characteristics of level 3 (classification).

Keywords: geometric thought, module of learning of rotations, Van Hiele model.

\section{Rotaçóes e níveis de raciocínio, de acordo com o modelo de Van Hiele: resultados de uma experiência}

\section{ResUmo}

Este artigo apresenta os resultados de uma pesquisa qualitativa do desenho etnográfico, realizada com oito (8) estudantes de quinta série do ensino secundario da Instituição Educacional Fernando Belaúnde Terry de Ate, com o objetivo de conhecer o desenvolvimento do pensamento geométrico dos estudantes nas rotaçóes, de acordo com o modelo de Van Hiele. A coleta de dados foi realizada por meio do acompanhamento do desenvolvimento das atividades programadas no módulo de aprendizado de rotaçóes elaborado de acordo com o modelo de Van Hiele, o teste de formaçáo de respostas abertas e a entrevista mista. Os resultados mostram que a maioria dos alunos exibiu características de nível 2 (análise) com um bom caminho para a aquisição de características do nível 3 (classificação).

Palavras-chave: pensamento geométrico, módulo de aprendizagem de rotaçóes, modelo de Van Hiele. 


\section{INTRODUCCIÓN}

El problema de la enseñanza de la matemática siempre ha sido un tema crucial para los docentes. Al respecto, estudios como el de Hernández y Villalba (como se citó en Vargas y Gamboa, 2013) indican que, «en los cursos de geometría, se presenta al estudiante un producto final y ya terminado, lo cual no da lugar a que él tome un papel activo en el desarrollo de su conocimiento matemático; además, no propicia el fomento de la creatividad y del aprendizaje significativo en el estudiante» (p. 76).

No obstante, los docentes siempre han mostrado preocupación para mejorar la didáctica y conducir a sus estudiantes a un buen aprendizaje de la geometría. Tanto es así que los esposos Pierre Van Hiele y Dina Van Hiele-Geldof, por la década de 1950, se preocuparon porque sus estudiantes no entendían lo que se les explicaba y realizaron una investigación, con los mismos, sobre el desarrollo del pensamiento geométrico y su contribución en la mejora de la calidad de sus razonamientos.

Bajo estos acontecimientos y siendo un tema que atañe a nuestra sociedad actualmente, el presente trabajo de investigación se basa en la didáctica de la geometría y forma parte de los resultados obtenidos en una investigación llevada a cabo para culminar la tesis de doctorado. El presente informe aborda la investigación que estuvo dirigida a la enseńanza de la geometría y al aprendizaje de los estudiantes del $5^{\circ} \mathrm{B}$, VII ciclo de la Educación Básica Regular (EBR) de la institución educativa (IE) Fernando Belaúnde Terry del distrito de Ate en la ciudad de Lima (Perú), del programa Jornada Escolar Completa, en torno al desarrollo del pensamiento geométrico sobre movimientos rígidos en el plano de transformaciones geométricas, mediante la aplicación de un módulo de aprendizaje organizado con actividades diseńadas bajo el modelo de Van Hiele y el cumplimiento de las fases de aprendizaje para una didáctica adecuada de la geometría, a fin de facilitar el razonamiento geométrico en los estudiantes en el tema en estudio.

\section{Marco teórico}

\subsection{Pensamiento geométrico}

Según Musser y Burger (1996), (como se citó en Gutiérrez, s/f), sobre el pensamiento geométrico, han propuesto describirlo a través de niveles de madurez que se van alcanzando de manera graduada, de acuerdo al avance en la estructura del conocimiento. Para ello, propone cinco niveles: nivel 0, visualización; 
nivel 1, descripción; nivel 2, de relaciones; nivel 3, de deducción; y, finalmente, nivel 4, de axiomatización. Estos niveles se van dando paulatinamente en el estudiante y requieren de un escenario didáctico que los favorezca.

Asimismo, sobre el desarrollo del razonamiento, Jaime y Gutiérrez (1990) exponen lo siguiente:

En general, el proceso de desarrollo del razonamiento no puede enmarcarse en los límites de un curso escolar. La adquisición de los niveles superiores, en particular del 3 y el 4 suele ser un proceso de varios ańos, por lo que no es de extrañar que al terminar el curso los estudiantes sigan estando en el mismo nivel que al principio, si bien estarán más cerca de poder lograr el nivel superior (p. 337).

\subsection{Módulo de aprendizaje}

Martínez-Salanova (s/f) expone en su blog que «según Bruner, la teoría de la Instrucción se ocupa de prescribir sobre la forma como debemos encarar la enseñanza para lograr un mejor aprendizaje» (párr. 2). Bruner, citado por Martínez-Salanova, sostiene que se ha tomado el concepto de módulo de instrucción para definir al módulo de aprendizaje. Al respecto, Yukavetsky (2003) afirma que «un módulo instruccional es un material didáctico que contiene todos los elementos que son necesarios para el aprendizaje de conceptos y destrezas al ritmo de la estudiante y sin el elemento presencial continuo del instructor» (p. 1). En este sentido, un módulo de aprendizaje es la colección de las actividades presentadas por la docente para cada una de las fases de aprendizaje.

\subsection{Guías de instrucción programada}

Heinz y Schiefelbein (2003) indicó que «en la instrucción programada, el alumno adquiere (autónoma e individualmente) conocimientos y habilidades (establecidos previamente) con la ayuda de textos programados en pequeños pasos (etapas) de aprendizaje» (p. 91).

\subsection{El modelo de Van Hiele}

Fouz (2005), a partir de algunas ideas previas al modelo y referidas a los estudiantes, basadas en la experiencia del trabajo del matrimonio Van Hiele, señala lo siguiente:

El aprendizaje de la Geometría se hace pasando por unos determinados niveles de pensamiento y conocimiento», «que no van asociados a la edad» y «que 
solo alcanzado un nivel se puede pasar al siguiente» [...]. Podemos señalar entre otras que, en la base del aprendizaje de la Geometría, hay dos elementos importantes «el lenguaje utilizado» y "la significatividad de los contenidos». Lo primero implica que los niveles, y su adquisición, van muy unidos al dominio del lenguaje adecuado y, lo segundo, que solo van a asimilar aquello que les es presentado a nivel de su razonamiento. Si no es así, se debe esperar a que lo alcancen para enseńarles un contenido matemático nuevo (pp. 67-68).

\subsubsection{Características del modelo}

Gutiérrez y Jaime (1998) indican que el modelo es descriptivo y prescriptivo. Por un lado, es descriptivo en tanto que intenta explicar cómo razonan los estudiantes. Esto se hace a través de la definición de cinco niveles de razonamiento. Por otro lado, es prescriptivo porque da unas pautas a seguir en la organización de la enseñanza para lograr que los estudiantes progresen en su forma de razonar. Esto se lleva a cabo mediante la consideración de cinco fases de aprendizaje.

\subsubsection{Propiedades del modelo de Van Hiele}

Sanz (2001) (como se citó en Blanco, 2015) sostiene que el modelo de Van Hiele contiene las siguientes propiedades:

Secuencial: Una persona debe recorrer los niveles en orden. Para tener éxito en un nivel el estudiante tiene que haber adquirido las estrategias de los niveles precedentes.

Progresivo: El progreso de un nivel a otro depende más del contenido y métodos de instrucción que de la edad.

Intrínseco y extrínseco (explícito/implícito): Los objetos inherentes (o implícitos) en un nivel pasan a ser objetos de estudio explícitos en el nivel siguiente.

Lingüístico: Cada nivel tiene sus propios símbolos lingüísticos y sus propios sistemas de relaciones entre símbolos.

Desajuste: Si el profesor, los materiales empleados, el contenido, el vocabulario, etc. están en un nivel superior al del estudiante, este no será capaz de comprender lo que se le presente y no progresará (p. 2).

\subsubsection{Niveles de razonamiento geométrico de Van Hiele}

El modelo de Van Hiele ayuda a explicar cómo, en el proceso de aprendizaje de la geometría, el razonamiento geométrico de los estudiantes transcurre por una serie de niveles. Para dominar el nivel en que se encuentra y así poder pasar al nivel inmediato superior, el estudiante debe cumplir ciertos procesos de logro y aprendizaje (Vargas y Gamboa, 2013, p. 81). 
Al respecto, Aravena y Caamaño (2013) expresan a detalle cada uno de dichos niveles:

Nivel 1. Reconocimiento: Es el nivel más elemental de razonamiento, los estudiantes perciben las figuras geométricas en su totalidad, pudiendo incluir atributos irrelevantes en las descripciones que hacen.

Nivel 2. Análisis. Es en este nivel donde se presenta por primera vez un tipo de razonamiento, que podría llamarse matemático. Los estudiantes son capaces de descubrir y generalizar propiedades, a partir de la observación y la manipulación.

Nivel 3. Clasificación: En este nivel los estudiantes pueden entender que unas propiedades pueden deducirse de otras y adquieren la habilidad de conectar lógicamente diversas propiedades de la misma o de diferentes figuras.

Nivel 4. Deducción formal: El estudiante logra la capacidad de razonamiento lógico matemático y una visión globalizadora del área que se esté estudiando (p. 149).

\subsubsection{Fases de aprendizaje en el modelo de Van Hiele}

Por otro lado, Gutiérrez y Jaime (como se citó en Lobo, 2004) detallan cinco fases del modelo que le competen al docente de la siguiente manera:

Fase 1 información: $\mathrm{Al}$ iniciar el estudio de un tema, el profesor informa sobre el campo de investigación a trabajar, los problemas a resolver e indaga los conocimientos previos y el nivel de razonamiento del grupo.

Fase 2 orientación dirigida: Los estudiantes exploran el campo de investigación mediante una serie de actividades dirigidas al descubrimiento y aprendizaje de los conceptos y propiedades fundamentales del área de estudio, para ello se han diseñado actividades de instrucción programada que conforman el módulo de aprendizaje.

Fase 3 explicitación: Se basa en el diálogo entre los estudiantes con intervenciones del profesor cuando sea necesario, a fin de conseguir que las experiencias adquiridas se unan a los símbolos lingüísticos precisos dentro de las características del nivel de razonamiento respectivo.

Fase 4 orientación libre: Los estudiantes aplican sus nuevos conocimientos a investigaciones posteriores sobre el tema de estudio, para ello se asignan tareas que preferiblemente lleven a diferentes soluciones.

Fase 5 integración: El profesor resume el campo explorado con la finalidad de lograr que los estudiantes integren en su red de conocimientos las habilidades de razonamiento adquiridas. (p. 4) 


\subsubsection{Descriptores generales evaluados de los niveles de Van Hiele en Rotaciones}

Basado en los descriptores generales de los niveles de Van Hiele, Gutiérrez, Jaime y Fortuny (1991), quienes organizaron un listado inicial de los descriptores específicos, se adaptó los descriptores o procesos claves para los niveles de las rotaciones, que a continuación se detalla.

- Nivel 1 reconocimiento: Identifica una figura por su forma y apariencia, clasifica recortes de figuras, no comprende las propiedades de las figuras. A partir de ejemplos de arquitectura, en este caso figuras geométricas de la cultura Inca, arte de la cultura árabe y de Escher. El estudiante indica la presencia de elementos geométricos rotados y comprende, de manera empírica, que la rotación preserva distancias y el ángulo de rotación y demuestra, de manera física, propiedades de isometría. En este nivel, el estudiante identifica que dos figuras rotadas en el plano son congruentes, pero no es capaz de conocer o explicar propiedades de la figura y no precisa el lenguaje básico geométrico. Puede mencionar el cambio de posición o expresar «ha girado» en vez de utilizar la terminación «rotación» sin identificar las condiciones de la misma.

- Nivel 2 análisis: El objetivo de este nivel es establecer los elementos necesarios para caracterizar la isometría, identificando y analizando partes y propiedades particulares de una figura rotada, y percibir los componentes y propiedades de la rotación (condiciones necesarias). De manera informal, se puede describir la rotación por sus propiedades, pero no ser capaz de relacionar unas propiedades entre sí. Puede establecer relaciones entre las figuras u objetos a través de la manipulación experimentación, pero no se puede realizar clasificaciones de figuras a partir de sus propiedades, ni deducir propiedades, (tomadas como premisas) de otras, porque se percibe cada una de ellas en forma aislada pero no se puede relacionar con las demás. Esta demostración es netamente empírica y de observación, ya que el estudiante no es capaz de elaborar definiciones, pero sí entenderlas con una estructura sencilla.

Los estudiantes identifican, por experimentación, que una rotación es obtenida rotando una figura en torno a un punto de giro. En este momento, se desea que los estudiantes puedan identificar los elementos que componen la rotación de forma aislada: un punto por el cual gira la figura y un ángulo que determina un giro. Del mismo modo, pueden probar en algunos puntos de la figura rotadas para identificar el centro de rotación como mediatriz y el punto del centro de rotación a la imagen para comprobar la igualdad de distancias entre cualquier punto y su respectiva imagen. 
Jaime y Gutiérrez (1990) afirman que "para un estudiante del nivel 2, demostrar consiste, simplemente, en comprobar que la afirmación es cierta en unos pocos casos, incluso en uno solo, haciendo mediciones oportunas con alguna herramienta. Esto le bastará para aceptar la veracidad de la afirmación» (p. 313).

- Nivel 3 clasificación: Consigue construir interrelaciones entre las figuras, reconociendo propiedades que derivan de otras. Las definiciones (condiciones necesarias y suficientes) adquieren significado, pero el razonamiento lógico sigue basado en la manipulación. Asimismo, el alumno no comprende el sistema axiomático de las matemáticas, solo sigue demostraciones, aunque no pueda entenderlas en globalidad, por lo que no organiza secuencias de razonamiento lógico y solo pueden llevar a cabo las inferencias simples por sí mismas, pero el razonamiento lógico sigue basado en la manipulación.

- Nivel 4 deducción formal: Aquí, el nivel de precisión que el estudiante tiene constituye una condición que le permite estudiar, comprender y aplicar los procesos de la matematización, Es decir, comprenderá la elaboración de algunos procesos de matematización. Realiza deducciones y demostraciones lógicas y formales, comprende y maneja las relaciones entre propiedades, entiende la naturaleza axiomática de las matemáticas, realiza pruebas formales matemáticas y entiende que a través de diferentes demostraciones puede llegar a los mismos resultados. Del mismo modo, los estudiantes matematizan situaciones problemáticas como parte del proceso de solución del problema final y contribuyen a la elaboración y reconocimiento de las partes rotadas mediante el cumplimiento de propiedades demostradas.

\subsection{Rotaciones}

En su obra, Wagner (1993) expuso:

Fijemos un punto $\mathrm{O}$ en el plano $\prod$ ahora orientado (como la tradición recomienda, la dirección positiva es anti-horaria). Dado un ángulo $\alpha$, la rotación de centro $\mathrm{O}$ y la amplitud $\alpha$ es la transformación que a cada punto A del plano $\prod$ asocia el punto $=(\mathrm{A})$ de modo que se tiene el segmento $\mathrm{O}=$ al segmento $\mathrm{OA}$, el ángulo $\mathrm{AO}=\alpha$ y el sentido del punto $\mathrm{A}$ para el punto (alrededor de O), positivo (figura 1).

Para cualquier $\mathrm{k}$ entero, las rotaciones de amplitudes $\alpha+360^{\circ}$ son idénticas. En particular, para $0^{\circ} \leq \alpha \leq 360^{\circ}$, la amplitud de rotación $-\alpha$ es igual a amplitudes de rotación $360^{\circ}-\alpha$. Una rotación es una isometría y, por tanto, transforma recta en rectas. 


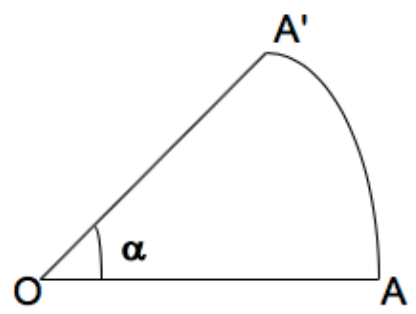

Figura 1. Rotación del segmento OA mediante el ángulo $\alpha$

Se define, entonces: sea un punto $\mathrm{O}$ del plano y un ángulo orientado $\alpha$, el giro de centro $\mathrm{O}$ y un ángulo $\alpha$ es una aplicación del plano en sí mismo $\mathrm{C}(\mathrm{O}, \alpha)$ : $\Pi$ $\rightarrow \prod$ tal que: $\mathrm{G}(\mathrm{O}, \Pi)(\mathrm{A})=\mathrm{A}^{\prime}$ si y solo si $\mathrm{d}(\mathrm{O}, \mathrm{A})=\mathrm{d}\left(\mathrm{O}, \mathrm{A}^{\prime}\right)$ y $\Varangle \mathrm{AOA}^{\prime}=\alpha$, para todo $A$ perteneciente a $\Pi$. Toda simetría central es una isometría (p. 72).

\subsection{Isometrías}

Respecto a las isometrías, Coxeter y Greitzer (1967) explicaron lo siguiente:

En particular, la geometría euclidiana se caracteriza por el grupo de similitudes; estas son transformaciones que preservan el ángulo. Un caso especial importante de una similitud es una isometría. Esta es una transformación de preservación de longitud tal como una rotación o, en particular, un medio giro. Las isometrías están en la parte inferior de la idea familiar de congruencia: dos figuras son congruentes si y solo si uno puede ser transformado en el otro por una isometría (p. 80).

\subsection{Evaluación formativa}

La Agencia de Calidad de la Educación (s/f) indica que:

La evaluación formativa se basa en un proceso de evaluación continuo que ocurre durante la enseńanza y el aprendizaje, basado en la búsqueda e interpretación de evidencia acerca del logro de los estudiantes respecto a una meta. Esto permite que el docente pueda identificar dónde se encuentran los aprendices, conocer qué dificultades enfrentan en su proceso de aprendizaje y determinar qué es lo que sigue y hacia dónde deben dirigirse para cerrar la brecha entre el conocimiento actual y las metas esperadas y cuál es el mejor modo de llegar hasta ahí (párrafo 3). 


\section{Metodología}

El objetivo de la investigación fue examinar cómo es el desarrollo del pensamiento geométrico y el progreso de los niveles de Van Hiele, en el grupo experimental de estudiantes del VII ciclo de la EBR, de la IE Fernando Belaúnde Terry de Ate, aplicando actividades propuestas en el Módulo de Aprendizaje Transformaciones Geométricas, Sección Rotación, para ubicar los niveles de Van Hiele correspondientes en la prueba formativa y determinar el logro de aprendizaje.

La metodología de trabajo fue cualitativa, desde un paradigma interpretativo de corte transversal. La perspectiva epistemológica básica desde la cual se realiza la indagación se refiere a un diseño etnográfico escolar y es la que ha direccionado la investigación y ha guiado el tipo de instrumento y técnicas a utilizar, los que sirvieron de sustento para atender al problema planteado. Para evidenciar el progreso de los niveles de Van Hiele, se complementó con otro instrumento adicional de la investigación cuantitativa, como fue la prueba de comprobación de respuestas abiertas, permitiendo, de esta manera, medir el progreso de los niveles de Van Hiele y responder a la pregunta de investigación. La metodología empleada fue adecuada de acuerdo a los conceptos de los siguientes autores:

Rodríguez, Gil y García (1999), en su obra, exponen:

Como quinta condición nos encontramos con la utilización de instrumentos en el proceso de realizar una etnografía. Los registros de las observaciones y de las entrevistas son la base del trabajo etnográfico, pero pueden utilizarse otros instrumentos tales como cuestionarios.

La utilización de la cuantificación cuando sea necesario se constituye en la sexta condición de una etnografía educativa. La cuantificación no es el punto de inicio, ni el objetivo último. Tanto la instrumentación como la cuantificación han de entenderse como procedimientos empleados para reforzar ciertos tipos de datos, interpretaciones o comprobación de hipótesis de distintos grupos (p. 46).

Asimismo, Gürtler y Günter (2007) mencionan que Los trabajos de Barton y Lazarsfeld (citados por Erzberger y Kelle, 2003) provocan un cambio importante de la investigación cualitativa. Por su función de «estudio previo», para posteriores estudios cuantitativos (vea también Mayring, 2001), la investigación cualitativa recibe aprecio como instrumento importante de investigaciones científicas. 


\subsection{Técnicas e instrumentos}

Las técnicas para recoger la información del diseño etnográfico escolar que se aplicaron fueron: la encuesta, la observación y la entrevista semiestructurada (mixta) y sus correspondientes instrumentos: el cuestionario, la ficha de observación y el guion de entrevista.

Las técnicas para recoger los datos y medir los niveles de razonamiento fueron las siguientes: una evaluación de entrada, una evaluación formativa y una entrevista semiestructurada (mixta). Los instrumentos utilizados fueron: una prueba de entrada, una prueba de comprobación y un guion de entrevista mixta. La prueba de comprobación fue la misma que la prueba de entrada, en virtud de que, en la prueba de entrada, los estudiantes demostraron no conocer el tema ni demostrar la adquisición de los niveles esperados en rotaciones. Asimismo, sobre los reactivos, se les asignó el mayor peso a los reactivos que contienen el nivel 3 porque es el nivel deseado por los docentes al culminar el $5^{\circ}$ de secundaria, y se consideró tres reactivos y otra de opción de niveles 2 o 3 . Respecto al nivel 2, cuyas características generalmente son adquiridas por los estudiantes de $5^{\circ}$ de secundaria, se le otorgó mayor peso que al nivel 1 . En el nivel 4, se consideraron dos reactivos para comprobar si habían incursionado en la abstracción y el método axiomático y se demostró que sus características no fueron adquiridas en su mayoría. Los puntajes ponderados de cada nivel se suman para obtener el puntaje total, como se aprecia en la tabla 1 .

\section{Tabla 1. Matriz de instrumento de recolección de datos de la prueba de entrada formativa}

\begin{tabular}{lcccl}
\hline Niveles de Van Hiele & $100 \%$ & Peso: 20 & No de reactivos/ puntaje & Instrumento \\
\hline Nivel 1 & $10 \%$ & 2 & $1(2)$ & \\
Nivel 2 & $20 \%$ & 4 & $2(2)$ & Cuestionario \\
Nivel 3 & $30 \%$ & 6 & $3(2)$ & de respuestas \\
Nivel 4 & $20 \%$ & 4 & $2(2)$ & abierta y una \\
Nivel 2 y 3 & $10 \%$ & 2 & $1(2)$ & \\
Nivel 3 y 4 & $10 \%$ & 2 & $1(2)$ & \\
\hline
\end{tabular}

Fuente: Elaboración propia, basada en los procesos de razonamientos clave de Gutiérrez y Jaime (1998, p. 32).

- La prueba de entrada y de comprobación fue un cuestionario con diez reactivos de respuestas libres, que dieron lugar a justificaciones o comentarios. La tabla 2 muestra los reactivos y sus correspondientes niveles de 
razonamiento. Se aprecia, del mismo modo, que hay reactivos que tienen hasta dos niveles de razonamiento. Esto se debe a que un mismo reactivo puede contener las características de los procesos de razonamiento clave que le compete a dos niveles o más.

Tabla 2. Reactivos y niveles de razonamiento de la prueba formativa de Rotaciones

\begin{tabular}{ll}
\hline Reactivos $\mathrm{N}^{\circ}$ & Niveles de razonamiento \\
\hline 9 & 1: Reconocimiento \\
1,8 & 2: Análisis \\
2 & 2 y 3: Análisis y clasificación \\
4,6 y 10 & 3: Clasificación \\
7 & 3 y 4: Clasificación y deducción formal \\
3,5 & 4: Deducción formal \\
\hline
\end{tabular}

Nota: En la elaboración de los reactivos se ha tenido en cuenta las características de los niveles de razonamiento en cada nivel de Van Hiele y sus procesos claves correspondientes.

La evaluación del cuestionario de respuestas libres siguió las pautas del trabajo de investigación de Jaime y Gutiérrez (1993), quienes indican que un ítem de respuesta libre puede ser contestado en distintos niveles de Van Hiele [...]. Esta variedad de posibilidades hace que, a la hora de evaluar una respuesta, primero se deba determinar el nivel de razonamiento en el que se ha respondido y después se deba analizar la calidad de la respuesta de la perspectiva del nivel que se considera, teniendo en cuenta tanto su precisión matemática como el empleo del nivel de razonamiento en cuestión.

Este doble análisis de las respuestas (de nivel de razonamiento y de corrección matemática) lleva a establecer siete tipos de respuestas, de las cuáles solo emergen en este trabajo los siguientes tipos:

Tipo 5. Respuestas bastante completas, pero matemáticamente incorrectas, que reflejan claramente la utilización predominante de un nivel de razonamiento. La incorrección de las respuestas pudo deberse a errores matemáticos o que siguen una línea de trabajo que no lleva a la solución del problema planteado, pero cuyos procesos de razonamiento son válidos.

Tipo 6. Respuestas bastante completas y matemáticamente correctas que reflejan claramente la utilización predominante de un nivel de razonamiento determinado. Se trata de respuestas claras y correctas, pero que no están completas porque no llegan a resolver el problema totalmente, porque hay «saltos» en el razonamiento deductivo seguido, porque tiene pequeños errores, etc. 
Tipo 7. Respuestas matemáticamente correctas y completas que reflejan claramente la utilización de un nivel de razonamiento determinado. La ponderación de cada tipo de respuesta guarda relación con los intervalos del segmento $[0,100]$, fijados para los grados de adquisición de los niveles. Como se muestra en la tabla 3.

Tabla 3. Ponderación de los diferentes tipos de respuesta

\begin{tabular}{lccccccc}
\hline Tipo & 1 & 2 & 3 & 4 & 5 & 6 & 7 \\
\hline Ponderación $(\%)$ & 0 & 20 & 25 & 50 & 75 & 80 & 100 \\
\hline
\end{tabular}

Fuente: Jaime y Gutiérrez (1993, p. 269).

Asignada la ponderación, se ubica el porcentaje en un intervalo porcentual, para fijar los grados de adquisición de los niveles, como se muestra en la tabla 4.

Tabla 4. Limites razonables para los diferentes grados de adquisición

\begin{tabular}{lc}
\hline Grados de adquisición & Porcentajes \\
\hline Nula & $0 \% \leq \mathrm{Gr} \leq 15 \%$ \\
Baja & $15 \%<\mathrm{Gr}<40 \%$ \\
Intermedia & $40 \% \leq \mathrm{Gr} \leq 60 \%$ \\
Alta & $60 \%<\mathrm{Gr}<85 \%$ \\
Completa & $85 \% \leq \mathrm{Gr} \leq 100 \%$ \\
\hline
\end{tabular}

Fuente: Jaime y Gutiérrez (1993, p. 226).

- Un guion de entrevista para ser aplicado en la fase estructurada de la entrevista semiestructurada (mixta) a los estudiantes, cuyos resultados no fueron los esperados, siendo además una alternativa para descubrir respuestas libres encubiertas, lo que dio la posibilidad de la ubicación del nivel correspondiente que fue verificado in situ mediante ejemplos y contraejemplos propuestos espontáneamente en su fase no estructurada.

\subsection{Procedimiento}

El estudio de la Rotación inició con la observación participante. El investigador, para ser aceptado y ser parte de ellos, socializó con el grupo investigado entablando conversaciones de interés y preferencias de los estudiantes y así poder definir sus características y comportamientos que se deben tener en cuenta en el proceso enseñanza-aprendizaje, se tomó un interés especial en hacer participar a los estudiantes más callados. 
Gozó de observación directa a los estudiantes, durante el proceso de aprendizaje y en el análisis de discusiones colectivas que se producían en el equipo de trabajo, para observar el pensamiento geométrico, crítico y reflexivo, que brindaban los estudiantes a preguntas retadoras, teniendo en cuenta las fichas de observación y sus documentos personales de progreso de cada clase, plasmados en los cuadernos de campo e historias de vida. La acción participante es la segunda etapa del método hubo entrevistas mixtas cuando la evaluación formativa y sumativa no eran respondidas como se esperaba y en algunos casos cuando hubo ejercicios que no comprendía.

Finalizando el recojo de la información, se realizó la triangulación que constituyó el proceso básico para la validación de los datos en cuanto a las fichas de observación, el cuaderno de campo y fichas del participante.

Para el proceso de enseñanza-aprendizaje que aplica el Modelo de Van Hiele, se siguió el modelo cualitativo de Katayama (2014) que demandó aplicar una comparación constante, que abarque los siguientes aspectos:

- Comparación de datos y construcción de categorías, teniendo como base categórica el modelo de Van Hiele y sus cuatro categorías se compara resultados de entrevistas y resultados de los reactivos de ejercicios y de la prueba formativa.

- Contrastación de las categorías, se contrasta el nivel o categoría de Van Hiele con el desarrollo del pensamiento geométrico correspondiente a la descripción del proceso de actividades realizadas, respuestas de reactivos en evaluaciones y durante las entrevistas, donde se tuvo un cuidado especial el lenguaje matemático utilizado.

- Delimitación teórica, se da solo en los cuatro niveles de Van Hiele, puesto que son los únicos que le corresponden a la EBR, VII ciclo.

-Saturación, la saturación se refiere a dar respuesta, cuando ya no se tiene acceso a otros datos que contribuyen al logro del ascenso de la ubicación de los niveles de Van Hiele, teniendo presente que el avance de los niveles depende mucho de la práctica de actividades que realiza el estudiante y que se satura de la misma manera que se satura la teoría que se está desarrollando, es decir el cumplimiento de las propias características que posee cada nivel de Van Hiele para el paso de un nivel a otro.

En las sesiones de aprendizaje, previamente se dejaron tareas como buscar algunos conceptos relacionados con las rotaciones: sentido de rotaciones de ángulos, rotaciones de ángulos cuadrantales y otros, con el objetivo de que recuerden algunos conceptos que posiblemente conocían. A continuación, se recogió, de los estudiantes, los conocimientos previos y se les aplicó una prueba de entrada. Para llevar a cabo el desarrollo del pensamiento geométrico, se aplicó el Módulo de Aprendizaje de Rotaciones que incluye guías de 
instrucción programada, con actividades diseñadas de acuerdo a los niveles y fases de Van Hiele cuya finalidad era promover el alcance de un nuevo nivel en los estudiantes y el logro del aprendizaje del tema en estudio para el cual se aplicó la evaluación formativa. Las respuestas de las pruebas se clasificaron en diferentes niveles y para suministrar el grado de adquisición alcanzado por los estudiantes, se calculó la media aritmética de las ponderaciones asignadas a cada uno de los niveles asignados. Finalmente, ubicados los grados de adquisición (Gr) de la prueba de comprobación, se realizó un análisis estadístico descriptivo como lo es la media aritmética de los resultados obtenidos en dichas pruebas, de entrada y de salida o comprobación, para confrontar su evolución.

La investigación fue cualitativa porque se quiso comprender la razón de por qué los estudiantes no aprenden las Rotaciones. Así, Mejía (como se citó en Katayama, 2014) nos indica que «la investigación cualitativa estudia diferentes objetos para comprender la vida social del sujeto a través de los significados desarrollados por este [...]. Entre la principal fortaleza del método cualitativo está el que rescata al ser humano como centro de la reflexión, al tiempo que brinda una comprensión integral de este» (p. 43).

Con relación a la Geometría, Shaughnessy (como se citó en Goncalves, 2006) nos dice que:

Específicamente en el área de Geometría, es necesario que se sitúe a los alumnos como seres pensantes frente a su propio pensamiento y se promueva una autorreflexión sobre su saber matemático o, en este caso, sobre su saber geométrico, no como un saber estático, perfecto y cristalizado, sino muy al contrario, como un saber dinámico, imperfecto y nebuloso, es decir, como un saber en acción (p. 85).

El Módulo de Aprendizaje de Rotaciones fue un material didáctico que permitió a los estudiantes darles un saber en acción promoviendo la autorreflexión; en tanto que las entrevistas mixtas permitieron a la docente conocer la manera de pensar de los estudiantes y actuar en el instante.

\section{Resultados y Discusión}

A partir de la aplicación de las herramientas de investigación antes mencionadas, se presentan los siguientes hallazgos. La aplicación de la entrevista semiestructurada (mixta) permitió una mayor libertad y flexibiidad en la obtención de información. A continuación, en la figura 2, se da un ejemplo de entrevista semiestructurada (mixta): 
Profesora: ¿Por qué esta figura es una rotación?

Estudiante: Por qué tiene la misma forma y tamaño.

Profesora: entonces, ¿basta tener la misma forma y tamańo para que una figura sea considerada rotada?

Estudiante: creo que sí

Profesora: ¡creo? y ¿Cómo es una traslación? Podríamos considerar la traslación una rotación.

Estudiante: pero tiene la misma forma y tamaño, espere deme la regla voy a medir... los lados son iguales, tienen la misma forma y tamaño.

Profesora: sí. Pero la traslación es una rotación. ¿O no es? [...].

Figura 2. Fragmento de la ficha de registro de observación de la actividad. Identificando rotaciones

Como resultado de la entrevista, se verificó que muchos estudiantes pueden reconocer una rotación, pero no logran definirla, notándose que algunos de los estudiantes no comprenden que la rotación es un movimiento de cambio de orientación y que, dado un punto cualquiera del mismo, este permanece a una distancia constante del centro de rotación y confunden la rotación con la traslación, lo cual refleja el nivel de inmadurez geométrica del estudiante. En este diálogo, se pretendió desarrollar los elementos necesarios para un posible ascenso al nivel 2, pues no solo discute las propiedades de las figuras, sino que también lleva a relacionarlas entre sí. En esta entrevista, se observó que el docente insta al estudiante a producir esas relaciones con el lenguaje apropiado.

De las entrevistas semiestructuradas que fueron trabajadas entre el docente y el estudiante, después de algún tiempo y mucha discusión, el estudiante consiguió relacionar propiedades de la rotación, lo que permitió elaborar el concepto de rotación, descartando el concepto de traslación. Fue un momento productivo para el docente y el estudiante que razonaba en el nivel 1. Se plantearon preguntas direccionadas al reforzamiento del concepto de rotación, procurando valorar lo que se aplica al nivel correspondiente, como la observación directa de las figuras, las mediciones, la orientación de las figuras, etc.

Esta actividad exigió una gran atención del docente para mantener el nivel de comprensión de los estudiantes sobre los conceptos presentados. Las preguntas de la entrevista buscaron estimular la claridad de las ideas y de las respuestas convenientemente reformuladas para garantizar una resignificación de los conceptos tratados sin inhibir al estudiante. Este momento pareció apropiado para la introducción de un lenguaje más formal y, durante la discusión de algún punto dudoso, con mucho cuidado para no intimidar 
al estudiante y liberar la lengua y el raciocinio, se fue introduciendo términos propios de la geometría, como isometría, punto de rotación, etc.

Para verificar el progreso de los niveles de Van Hiele, se procedió a la interpretación y análisis de actividades desarrolladas en las fases de aprendizaje de cada uno de los niveles de Van Hiele, propuestas en el módulo de aprendizaje sección Rotaciones; como ejemplo, se presenta una actividad para cada fase de aprendizaje del nivel 2, se eligió el nivel 2 debido a que es el nivel de más alto porcentaje alcanzado:

Fase 1. Información. El factor más importante que influye en el aprendizaje es el conocimiento de lo que el estudiante sabe; por ello se indagó al respecto para que la docente oriente el aprendizaje en correspondencia con dicha indagación, proponiéndose las actividades siguientes:

Actividad 1, (Fase1, Nivel2).- Se realizó una presentación en Power Point con figuras de la cultura Paracas y del Alhambra, imágenes que fueron utilizadas como fuente de motivación y que permitieron conocer el grado de conocimiento y el nivel de razonamiento de los estudiantes respecto a las rotaciones, que fue evidenciado al responder los reactivos del material impreso, entregado posterior a la observación de la presentación en Power Point. A continuación, se presentan los reactivos y como ejemplo de respuestas, las que dio el estudiante $\mathrm{N}^{\circ} 6$.

1. Observa las figuras 3 y 4 , agrupe aquellas que piensa que son congruentes o iguales y que cambiaron de posición y explique por qué sucedió eso.

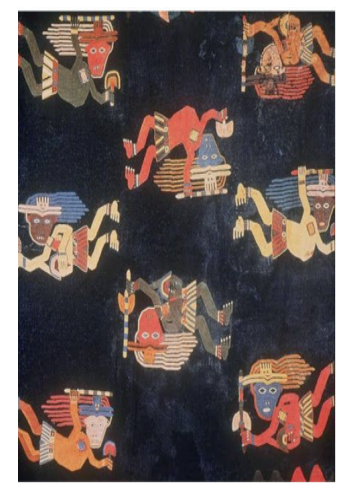

Figura 3. Manto fúnebre textilería de Paracas-Necrópolis de belleza. Fuente: https://www.pinterest.com/evelynehaun/moche/?lp=true 
Mercedes Maritza Sarrín Suárez

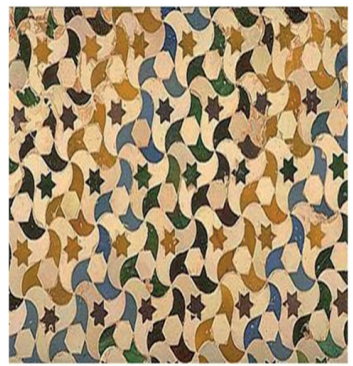

Figura 4. Pajarita Nazarí, figura base de un mosaico que podemos encontrar en la Alhambra de Granada.

Fuente:https://pruebadibujo.wordpress.com/2013/03/06/la-alhambra-escher-y-los-movimientos-en-el-plano/

\section{Respuesta}

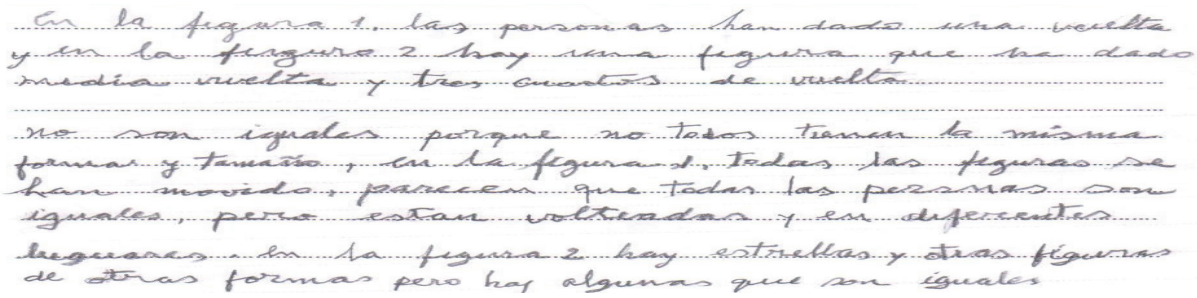

2. Agrupe aquellas que se parecen pero que crees que no son congruentes o iguales. indica ¿por qué? De acuerdo con la agrupación que se ha realizado, ¿cuántas figuras han rotado o girado?

\section{Respuesta}

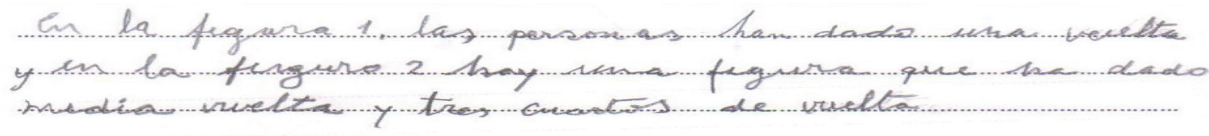

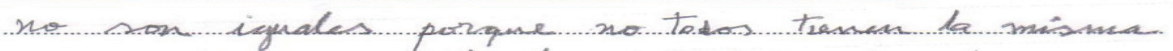

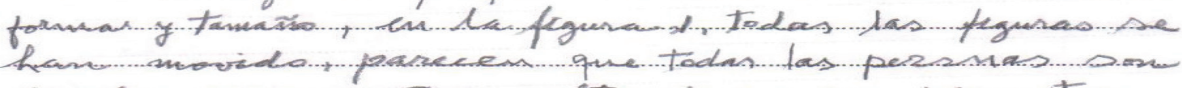

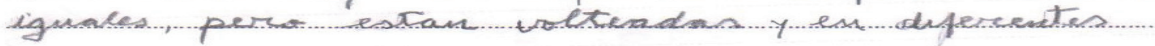

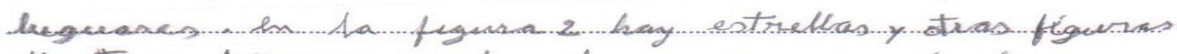
de derias formas pers har algumas que son iguales

Fase 2. Orientación dirigida, los estudiantes descubren y utilizan, como característica de las rotaciones, el centro y el ángulo de rotación. Deben saber determinar el ángulo de un giro, comprenden, aprenden y conservan los 
conceptos básicos y sus propiedades que, adicionalmente, relacionaron para la definición del concepto rotación siendo esta una condición necesaria para progresar del nivel 2 al nivel 3 de razonamiento del modelo educativo de Van Hiele.

Actividad 2 (Fase2, Nivel 2): El triángulo debajo del eje x se transforma en el triángulo rotado tras un giro de centro $\mathrm{C}$. Explica cómo se calcula el centro de giro y cómo son las medidas de las distancias del centro de giro y de los puntos homólogos obtenidos de la figura rotada.

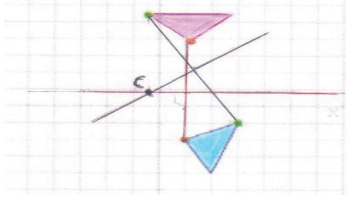

Figura 5. Actividad 3 de la Fase 2 del Nivel 2. Respuesta elaborada por la estudiante $\mathrm{N}^{\circ} 2$

\section{Respuesta}

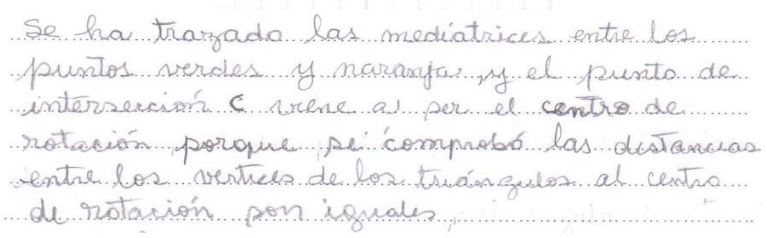

Fase 3. Explicitación: Dentro de un contexto de diálogo en un trabajo en equipo a través del uso del software Geo Gebra, se les pidió a los estudiantes que expliquen cómo han resuelto las actividades. Es la fase de revisión del trabajo en equipo desarrollado los estudiantes a partir de discusiones llegaron a distintas de conclusiones, donde se tomó en cuenta una de las características de los niveles, como es, el lenguaje.

Al respecto, Jaime y Gutiérrez (1990) señalan que «hay una estrecha relación entre el lenguaje $y$ los niveles. Las diferentes capacidades de razonamiento asociadas a los cuatro niveles de Van Hiele no solo se reflejan en la forma de resolver los problemas propuestos, sino en la forma de expresarse y en el significado que se le da a determinado vocabulario» (p. 313).

Las entrevistas semiestructuradas fue una técnica que accedió establecer el lenguaje y los niveles. 

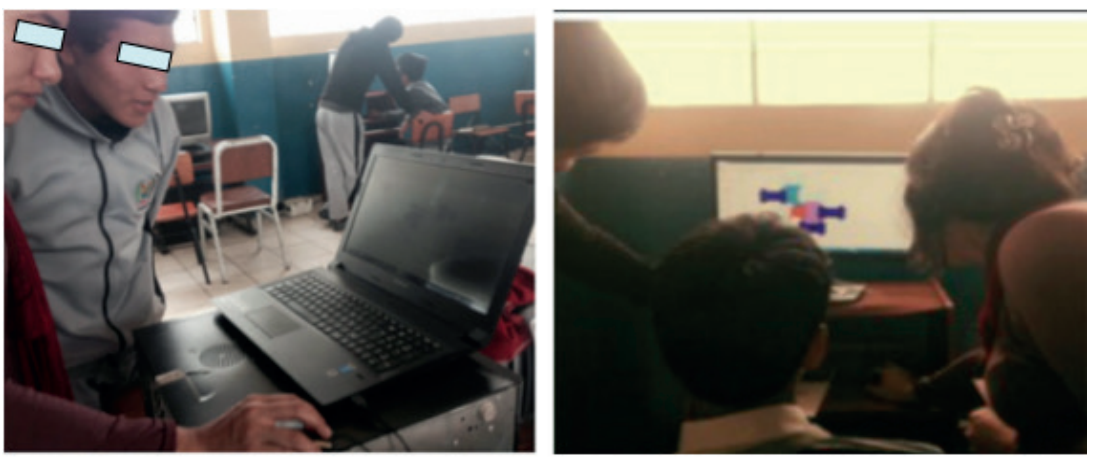

Figura 6. La docente escucha los términos y conceptos de la rotación, empleados por los estudiantes en la rotación de figuras efectuadas con el software Geo Gebra.

Nota: Fotos autorizadas por la profesora y los estudiantes para la investigación.

Fase 4. Orientación libre: Se aplica el conocimiento consolidado del aprendizaje realizado en las fases anteriores en una actividad compleja que plantee nuevas relaciones o propiedades, como el giro de una porción del triángulo que se muestra en la actividad propuesta. Sin la participación del docente, los estudiantes aplicaron sus conocimientos y el lenguaje matemático adquirido. En esta fase, se observó que el estudiante combina sus conocimientos y los aplica a situaciones diferentes a las anteriores para que, de lo aprendido en las fases, seguir el camino adecuado para la solución de la actividad.

Actividad 3 (Fase4, Nivel2): Observa el triángulo equilátero, ha sido modificado para formar la figura de contorno rojo. Explica cómo se ha formado dicha figura a partir del triángulo equilátero, ¿Crees que hubo rotación?, ¿Qué podrías decir acerca del área de la región interior de la figura modificada? Explica por qué sucedió eso.

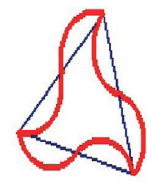

Figura 7. Tesela. Respuesta del estudiante $\mathrm{N}^{\circ} 1$. 


\section{Respuesta}

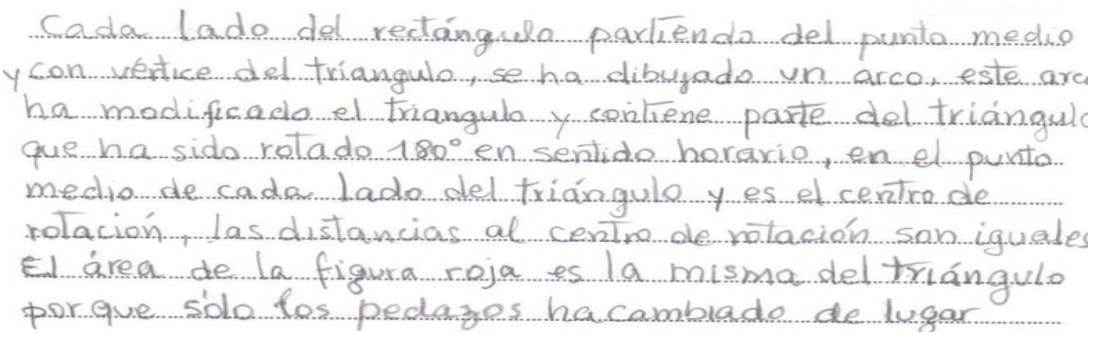

Fase 5. Integración: El módulo de aprendizaje sección Rotaciones, contempló actividades de comprensiones globales, de condensar en un todo el dominio que ha explorado el pensamiento de los estudiantes, como fue el pedir la construcción de un mapa conceptual del objeto de estudio.

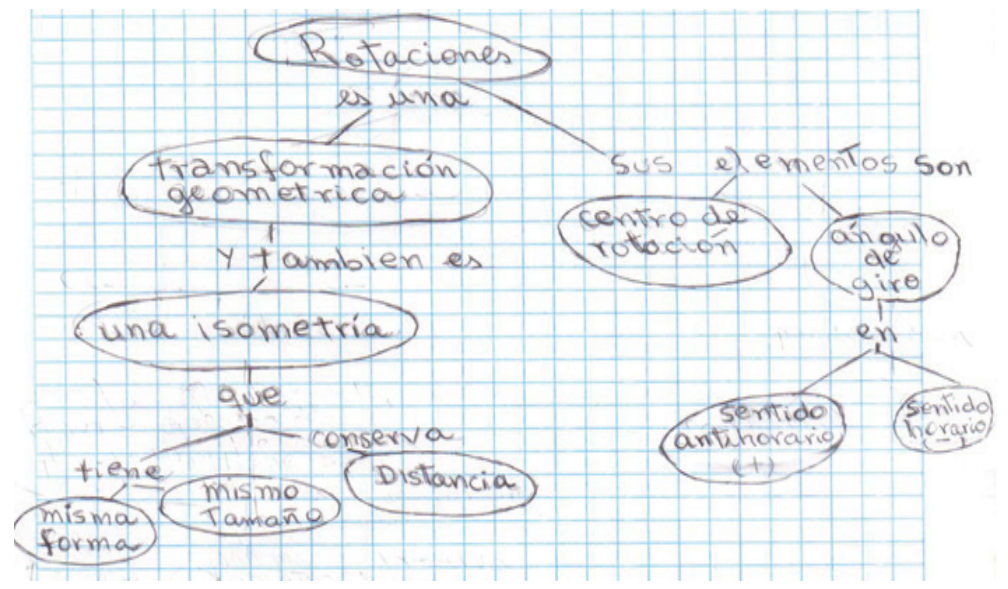

Figura 8. Mapa conceptual elaborado por la estudiante $\mathrm{N}^{\circ} 4$.

Por la naturaleza de esta fase, el mapa de la figura 8 muestra también la evolución del lenguaje utilizado. Por ejemplo, cuando utilizan el concepto isometría, se cumple con el requisito de integrar varios conceptos que originalmente aparecían desligados dentro del mismo: comprendieron que isometría es un concepto dinámico y que para elaborar razonamientos a partir del mecanismo de los elementos de la rotación deben hacerlo teniendo en cuenta que es su característica fundamental. Se corrobora lo expresado por Coxeter y Greitzer (1967), quienes dieron a entender que la figura es transformada por una isometría porque se observa que preserva longitud, y las figuras inicial y final son geométricamente congruentes. 
Para proporcionar la ubicación de los niveles de Van Hiele a los estudiantes, complementar la respuesta al problema planteado y dar cumplimiento al objetivo propuesto, se analizó las respuestas libres del cuestionario de diez (10) reactivos de la prueba formativa que proporcionaron los estudiantes; como ejemplo se ha tomado la respuesta que diera el estudiante $\mathrm{N}^{\circ} 1$ al reactivo $\mathrm{N}^{\circ}$ 8 , nivel 2 figura $\mathrm{N}^{\circ} 13$ y análisis del reactivo $\mathrm{N}^{\circ} 8$ del Nivel 2, figura 9.

Reactivo $N^{\circ} 8$. - La figura 9 muestra un hexágono regular inscrito en una circunferencia de centro o. Indique y justifique sus respuestas: a) Cuál es la imagen de $\mathrm{B}$ en una $\mathrm{R}\left(\mathrm{O}, 6^{\circ}\right)$ horaria; b) Cuál es la imagen de $\mathrm{C}$ en una $\mathrm{R}$ $\left(\mathrm{O}, 120^{\circ}\right)$ anti horaria; c) Cuál es la imagen del segmento DC en $\mathrm{R}\left(\mathrm{O}, 180^{\circ}\right)$ antihoraria.
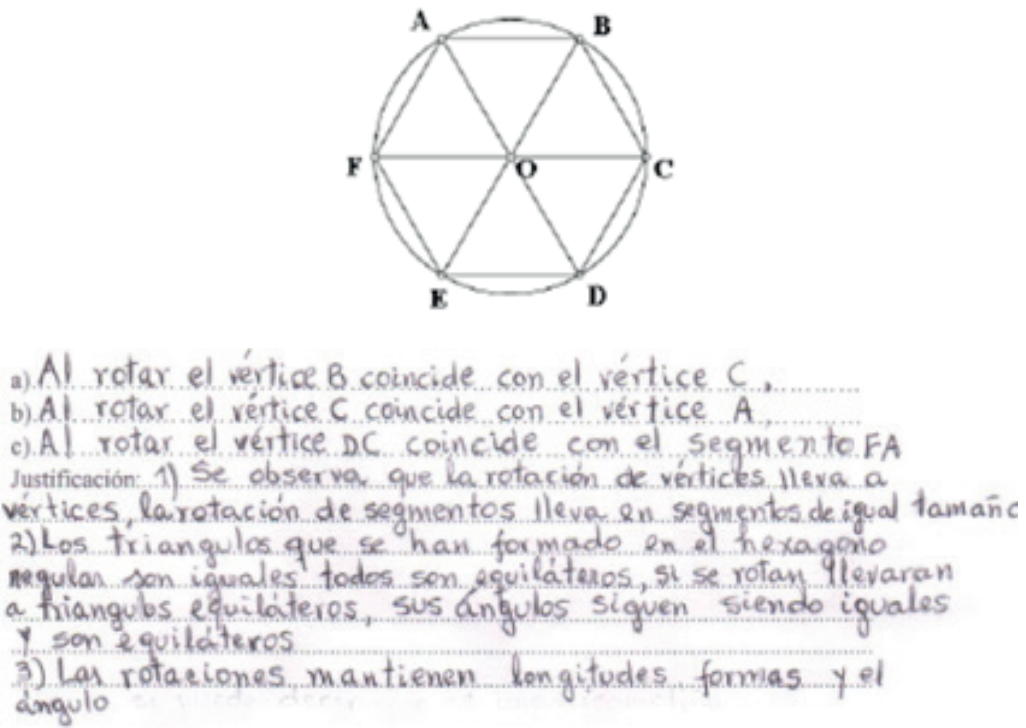

Figura 9. Ejemplo de respuesta del estudiante $\mathrm{N}^{\circ} 1$ en actividad del nivel 2.

Como lo mencionan en su obra, Jaime y Gutiérrez (1990) «Para determinar el nivel de razonamiento, lo más importante no es evaluar si los estudiantes contestan bien o mal, sino cómo contestan y por qué lo hacen así» (p. 321).

La entrevista semiestructurada (mixta) que permitió comprobar la forma de contestar de los estudiantes y por qué lo hacen así, admitió un razonamiento sobre el desarrollo de los ejercicios propuestos en las Guías de Instrucción Programadas contenidas en el Módulo de Aprendizaje de Rotaciones, donde el docente mediante preguntas retadoras ayudó a que los estudiantes construyan conceptos y procesos. 
Por último, se observan los resultados finales del reactivo $\mathrm{N}^{\circ}$ 8: Roberto, Rebeca, Yanira, Nataly Jordi y Joseph proporcionaron respuestas bastante completas, pero matemáticamente incorrectas. No recordaron el sentido del giro horario y antihorario, no identificaron que se trataba de un hexágono regular que está intimamente relacionado con los triángulos equiláteros que se formaron al unir cada vértice con su opuesto. Mientras tanto, Ervin obtuvo respuestas claras y correctas, pero no completas. Tiene pequeños errores como el de la respuesta c): confundió la rotación de $180^{\circ}$, no especificó que la imagen del segmento DC en $\mathrm{R}\left(\mathrm{O}, 180^{\circ}\right)$ es el segmento AF. Nilton se ubicó en el tipo 7 (ver tabla 5) matemáticamente correcta y completa. Ha reflejado claramente la utilización de un nivel de razonamiento determinado.

En la figura 10 se presentan los grados de adquisición alcanzados en el reactivo $\mathrm{N}^{\circ} 8$ de la prueba formativa de Rotaciones.

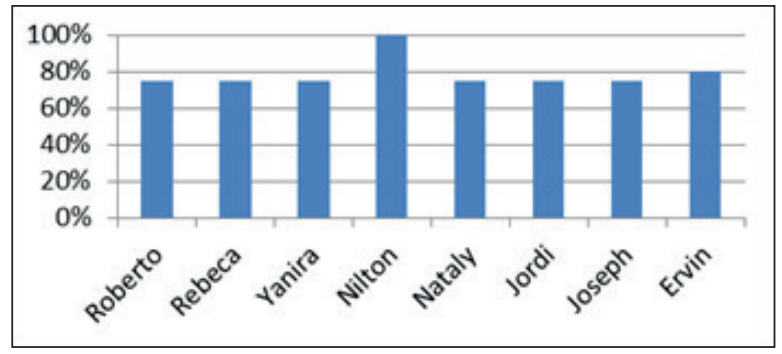

Figura 10. Grados de adquisición logrados en el reactivo $\mathrm{N}^{\circ} 8$

El Grado de adquisición promedio: G.A (Nivel 2)=78,6\%. Según la respuesta entregada por los estudiantes, las respuestas promedio del reactivo 8 correspondiente al Nivel 2, le corresponde un grado de adquisición del 79\%.

En conclusión, se observó que las actividades programadas permitieron resultados favorables, los estudiantes aun alcanzando un alto porcentaje en el nivel 2 progresaron en el logro de los aprendizajes.

El logro del nivel 3 implicaba la adquisición y significado de definiciones para establecer las condiciones necesarias y suficientes que deben cumplir las rotaciones, pero, debido a que el lenguaje utilizado en cada nivel no era asimilado oportunamente, no comprendían los enunciados de las actividades de los niveles y fases del aprendizaje, lo que desmotivaba al estudiante, haciéndoles perder el interés por comprender el tema. Los problemas de su entorno familiar y social como la violencia causada por el umbral de pobreza que le tocó vivir, la inasistencia e impuntualidad que ocasionalmente se producía, y de ser estudiantes poco comunicativos, interfirieron en el aprendizaje oportuno, por lo que 
Tabla 5. Descripción de la respuesta del reactivo $N^{\circ} 8$

\begin{tabular}{|c|c|c|c|c|}
\hline Estudiante & $\begin{array}{l}\text { Nivel de } \\
\text { VanHiele }\end{array}$ & Análisis de la respuesta & $\begin{array}{l}\text { Tipo de } \\
\text { respuesta }\end{array}$ & $\begin{array}{l}\text { Ponde- } \\
\text { ración } \\
(\%)\end{array}$ \\
\hline Roberto & 2 & $\begin{array}{l}\text { El hexágono tiene triángulos equiláteros } \\
\text { y sus ángulos son iguales a } 60^{\circ} \text {; por tanto, } \\
\text { Respuesta c) es el segmento AF porque } \\
60^{\circ}+60^{\circ}=120^{\circ} \text {, en a) en b) no consideró } \\
\text { la dirección de rotación tuvo error. Reflejó } \\
\text { claramente la utilización de las respuestas } \\
\text { con errores matemáticos, usó procesos de } \\
\text { razonamiento válidos. }\end{array}$ & 5 & 75 \\
\hline Rebeca & 2 & $\begin{array}{l}\text { No tuvo precisión en darse cuenta de la } \\
\text { medida de los ángulos, pero sí hubo indicios } \\
\text { de realizar las rotaciones con los puntos, no } \\
\text { tomó en cuenta que el hexágono está for- } \\
\text { mado por triángulos equiláteros y que por } \\
\text { consiguiente cada ángulo mide } 60^{\circ} \text {. No } \\
\text { respondió correctamente las preguntas a), } \\
\text { b) y c), la incorrección de las respuestas se } \\
\text { debe a errores matemáticos, sus procesos de } \\
\text { razonamiento son válidos. }\end{array}$ & 5 & 75 \\
\hline Yanira & 2 & Respuesta similar a la de Roberto. & 5 & 75 \\
\hline Nilton & 2 & $\begin{array}{l}\text { Dio las imágenes correctas justificando sus } \\
\text { movimientos y el tipo de ángulo formado. }\end{array}$ & 7 & 100 \\
\hline Nataly & 2 & $\begin{array}{l}\text { No considero el sentido de rotación en a) } \\
\text { por lo que tuvo error, completó correcta- } \\
\text { mente lo demás. }\end{array}$ & 5 & 75 \\
\hline Jordi & 2 & Respuesta similar a la de Rebeca. & 5 & 75 \\
\hline Joseph & 2 & $\begin{array}{l}\text { Tuvo un buen procedimiento pero confun- } \\
\text { dió el sentido del ángulo y tuvo errores, en } \\
\text { b) y c) completó correctamente. }\end{array}$ & 5 & 75 \\
\hline Ervin & 2 & $\begin{array}{l}\text { Respuesta clara y correcta pero con errores } \\
\text { matemáticos en la última pregunta, con- } \\
\text { fundió la rotación de } 180^{\circ} \text {. }\end{array}$ & 6 & 80 \\
\hline
\end{tabular}

el paso de un nivel de razonamiento a otro era lento y generaba tomar más tiempo en el logro del aprendizaje; la enseńanza-aprendizaje concordante con el nivel de su razonamiento y los conocimientos previos que traían se avanzó con paciencia, aun así no se enriquecían los aprendizajes al establecerse nuevos conocimientos ni se relacionaban entre ellos, porque a medida que iban avanzando los 
niveles, los descriptores eran de más exigencia y de mayor retención de términos y conceptos matemáticos dificultando el progreso al nivel 3 en su totalidad.

Al respecto la Universidad Internacional de Valencia (2015) menciona lo siguiente:

Existen factores internos, cuyo origen no es ni el propio alumno ni el sistema educativo, que también tienen una notable influencia: los factores de índole familiar y social. El marco social y familiar que envuelve al alumno ejerce un papel muy importante en la vida académica de los estudiantes, tanto directa como indirectamente. Son numerosos los estudios que demuestran las repercusiones de los factores familiares y sociales (clase social, nivel económico y cultural) en los resultados educativos, influyendo intensamente en el funcionamiento cognitivo del nińo y su motivación y, en consecuencia, teniendo un peso importante en su rendimiento educativo. La implicación familiar en la educación de sus hijos y la integración social influye positivamente en los logros académicos.

La comparación entre las Pruebas de entrada y formativa, en base al análisis estadístico descriptivo se observan en la tabla 6 , donde se obtuvo los siguientes resultados:

Tabla 6. Cuadro comparativo de resultados de la Prueba de entrada (PE) y la Prueba de salida o formativa (PS) de Rotaciones y los logrados de adquisición correspondientes

\begin{tabular}{|c|c|c|c|c|c|c|c|c|c|c|c|c|}
\hline \multirow[b]{2}{*}{ Estudiante } & \multicolumn{3}{|c|}{ Nivel 1} & \multicolumn{3}{|c|}{ Nivel 2} & \multicolumn{3}{|c|}{ Nivel 3} & \multicolumn{3}{|c|}{ Nivel 4} \\
\hline & $\begin{array}{c}\mathrm{PE} \\
\%\end{array}$ & $\begin{array}{l}\text { PS } \\
\%\end{array}$ & $\begin{array}{c}\text { Avance } \\
\%\end{array}$ & $\begin{array}{c}\text { PE } \\
\%\end{array}$ & $\begin{array}{l}\text { PS } \\
\%\end{array}$ & $\begin{array}{c}\text { Avance } \\
\%\end{array}$ & $\begin{array}{l}\text { PE } \\
\%\end{array}$ & $\begin{array}{l}\text { PS } \\
\%\end{array}$ & $\begin{array}{c}\text { Avance } \\
\%\end{array}$ & $\begin{array}{l}\text { PE } \\
\%\end{array}$ & $\begin{array}{l}\text { PS } \\
\%\end{array}$ & $\begin{array}{c}\text { Avance } \\
\%\end{array}$ \\
\hline Roberto & 20 & 100 & 80 & 0 & 41,6 & 41,6 & 0 & 33,3 & 33,3 & 0 & 0 & 0 \\
\hline Rebeca & 0 & 100 & 100 & 0 & 60 & 60 & 0 & 32,5 & 32,5 & 0 & 0 & 0 \\
\hline Yanira & 0 & 100 & 100 & 0 & 66,7 & 66,7 & 0 & 46 & 46 & 0 & 0 & 0 \\
\hline Nilton & 20 & 100 & 80 & 20 & 83,3 & 63,3 & 0 & 75 & 75 & 0 & 8,3 & 8,3 \\
\hline Nataly & 20 & 100 & 80 & 0 & 85 & 85 & 0 & 76 & 76 & 0 & 6,7 & 6,7 \\
\hline Jordi & 0 & 100 & 100 & 0 & 48,3 & 48,3 & 0 & 40 & 40 & 0 & 0 & 0 \\
\hline Joseph & 20 & 100 & 80 & 0 & 38,3 & 38,3 & 0 & 33,3 & 33,3 & 0 & 0 & 0 \\
\hline Ervin & 0 & 100 & 100 & 0 & 60 & 60 & 0 & 58,3 & 58,3 & 0 & 6,7 & 6,7 \\
\hline
\end{tabular}

Nota: La tabla muestra la comparación entre la PE (prueba de entrada) y la PS (prueba de salida o Prueba Formativa) del cuestionario de rotaciones y los grados de adquisición obtenidos por los estudiantes antes y después de la aplicación del módulo de aprendizaje sección rotaciones. Para el análisis de resultados de la prueba de entrada, se ha considerado solo cuatro de los diez reactivos propuestos, dado que los reactivos 2.3.4.6.7 y 8 no fueron respondidos por todos; en la prueba de salida, en general solo dos estudiantes respondieron satisfactoriamente el nivel 3, los demás dejaron en blanco o incompleta, el nivel 4 no salió satisfactorio. 
Nivel 1: Cuatro de ocho estudiantes (el 50\%), en la prueba de entrada, se encontraban con $20 \%$ de avance en el nivel 1. Esto demuestra que los estudiantes no indicaban la presencia de elementos geométricos rotados y no perciben que la rotación es una isometría. También no identificaban que, en el plano, dos figuras son congruentes. Finalmente, en la prueba de salida, se logró un $80 \%$ de avance que cubrió las expectativas.

Nivel 2: Siete de ocho estudiantes (el 87,5\%), en la prueba de entrada, se encontraban con $0 \%$ de avance en el nivel 2. Esto muestra que el estudiante no establecía los elementos necesarios para caracterizar las isometrías, no establecía relaciones entre las figuras y no identificaba que una rotación es obtenida girando una figura en torno de un punto de giro. En ese momento, se deseaba que los estudiantes puedan identificar los elementos que componen la rotación, un punto por el cual gira la figura y un ángulo que determina un giro. Si establecía relaciones entre figuras, habría podido acompañar una demostración formal acompañada de una verificación empírica. En los resultados de la prueba de salida, se observó que los grados de adquisición fueron: un 50\% en intermedia; un $25 \%$ en alta, un $12,5 \%$ en completa y un $12,5 \%$ en baja. Esto significó que el aula tuvo estudiantes con un logro entre destacado y previsto en el nivel 2.

Nivel 3: El total de los estudiantes no contaron con un avance en el nivel 3. Esto significa que, en las pruebas de entrada y salida, no consiguieron establecer relaciones entre las figuras; no pudieron acompañar una demostración formal cuando vivificaban una demostración empírica, no podían probar los puntos y sus homólogos de una figura para identificar el eje como mediatriz y el punto imagen de la distancia. Por tanto, no identificaban el punto de rotación y el eje de giro; es decir, no pudieron aplicar la propiedad a cualquier punto y su respectiva imagen. En la prueba final, se aprecia un $37,5 \%$ en el grado de adquisición baja, un $37,5 \%$ en intermedia y solo un $25 \%$ en alta. Esto indica que el nivel 3 ha logrado una ubicación baja, con un logro de aprendizaje considerable en inicio.

Nivel 4: El total de los estudiantes no contaron con un avance en el nivel 4, esto demuestra que el estudiante no adquirió el nivel de precisión y no adquirió condiciones para estudiar, comprender y aplicar los procesos de la mate matización completa. Es decir, comprendió solo la elaboración de solo algunos procesos de mate matización.

Este estudio indica que la mayoría de los estudiantes, antes de comenzar el estudio de la unidad de aprendizaje, no presentaban el nivel 1; es decir, mostraban un bajo nivel de reconocimiento a pesar que se les había dado tarea y algunos conceptos básicos de rotaciones, donde manifestaron que era la primera vez que veían este tema. 
A continuación, en la tabla 7 , se muestra el cuadro de consolidación, de cada estudiante, de los resultados de logros adquiridos del progreso en los niveles de Van Hiele después de aplicar la prueba formativa de Rotaciones y de los niveles alcanzados.

Tabla 7. Resumen de los niveles alcanzados en la prueba formativa de Rotaciones

\begin{tabular}{ccccc}
\hline Estudiante & nivel 1 & nivel 2 & nivel 3 & nivel 4 \\
\hline Roberto & completa & intermedia & baja & nula \\
Rebeca & completa & intermedia & baja & nula \\
Yanira & completa & alta & intermedia & nula \\
Nilton & completa & alta & alta & nula \\
Nataly & completa & completa & alta & nula \\
Jordi & completa & intermedia & intermedia & nula \\
Joseph & completa & baja & baja & nula \\
Ervin & completa & alta & intermedia & nula \\
\hline
\end{tabular}

Datos de la prueba formativa. Se aprecia un logro del nivel 1 en todos sus participantes, existiendo diferencias en el nivel 2 que solo un estudiante logró ubicarse en completa, dos en alta y tres en intermedio, dejando en estudio la situación de dos estudiantes en baja adquisición del nivel 2; en el nivel 3 , se aprecia que hay dos altas, tres intermedias y tres bajas; el nivel 4 fue en pleno nulo, los casos de ubicación baja de los niveles 2 y 3, tuvieron influencias de variables extrańas, que se pudo detectar mediante la entrevista no estructurada.

La ponderación obtenida del nivel 2 de Van Hiele en porcentajes de la prueba formativa de rotación en su conjunto se muestran en la figura 11. Esta figura muestra el resultado de mayor porcentaje adquirido por los estudiantes del grupo experimental donde se aprecia que, de las actividades de clasificación del nivel 2, hay un 60,40\% de logro que se ubica en el grado de adquisición intermedia. Cabe resaltar que, en las actividades últimas, se mostró afinidad en el tema de las rotaciones, a pesar de la importancia del lenguaje para el paso de un nivel a otro, en este caso, no fue bien concebido. Se usaron términos inadecuados, pero los estudiantes comprendían el concepto y la idea de rotación que, posteriormente, con la realización de las actividades, pudieron fijar el lenguaje, lo que les permitió continuar con la realización de las actividades de la rotación. En conclusión, se asignó a los estudiantes del grupo experimental, el nivel 2 logrado.

Por lo tanto, se cerró la etapa de reencuadre de las actividades consideradas de los niveles de Van Hiele. Fueron muchas las formas de tomar las fases de aprendizaje de Van Hiele. Posteriormente, para reforzar las respuesta no 
respondidas o incompletas se realizó la entrevista semiestructurada después de los resultados que arrojó la evaluación formativa sobre la ubicación de los niveles de Van Hiele, pero los estudiantes no se encontraban en su totalidad por lo que solo cinco pudieron participar en la entrevista mixta, siendo entrevistados en las condiciones en que se encontraban.

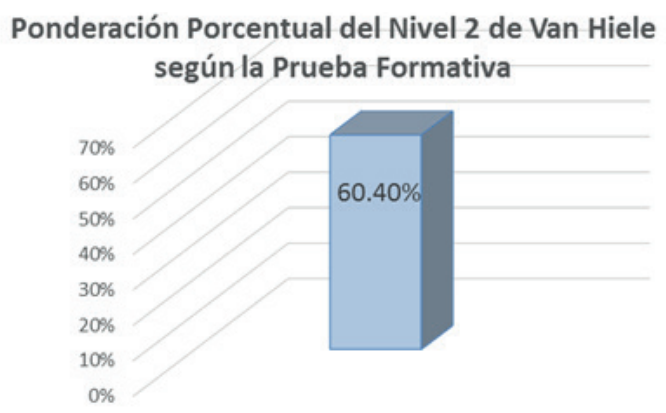

Figura 11. Ponderación Porcentual de logros alcanzados en el nivel 2 de Van Hiele según la prueba formativa de Rotaciones, del grupo experimental de la IE FBT. Diagrama de barra, que demuestran el progreso de los niveles de Van Hiele, alcanzados mediante la puesta en práctica de las actividades diseñadas en el Módulo de Aprendizaje Sección Rotaciones, que dieron como resultado una ubicación en el nivel 2, cabe resaltar que la ubicación no depende de la edad de los estudiantes, sino de las actividades que se realizó y fue un proceso lento.

Finalmente, en la tabla 8, se presenta un cuadro del reactivo que alcanzó el más alto porcentaje en el nivel 2, nivel que caracteriza el logro adquirido por el grupo experimental.

Tabla 8. Evidencia del reactivo de mayor cambio de la prueba diagnóstica en el nivel 2 de Van Hiele

\begin{tabular}{ccccc}
\hline Reactivo $\mathrm{N}^{\circ}$ & $\begin{array}{c}\text { PE } \\
\text { \% de logro }\end{array}$ & $\begin{array}{c}\text { PF } \\
\text { \% de logro }\end{array}$ & Nivel & $\begin{array}{c}\text { Tipo de respuesta } \\
\text { predominante }\end{array}$ \\
\hline 8 & 0 & 79 & 2 & 5 \\
\hline
\end{tabular}

La tabla muestra la comparación del reactivo $\mathrm{N}^{\circ} 8$ entre la PE (prueba de entrada) y la PF (prueba formativa) del cuestionario de rotaciones y el tipo de respuesta que predominó fue el 5: respuesta bastante completa pero matemáticamente incorrecta, reflejó claramente la utilización predominante del nivel de razonamiento 2, la incorrección de la respuesta se debió a errores matemáticos 
como el no recordar que el hexágono tiene triángulos equiláteros y sus ángulos son iguales a $60^{\circ}$, pero el proceso de razonamiento fue válido.

\section{Conclusiones}

- La metodología cualitativa facultó la observación y la entrevista semiestructurada (mixta) en la investigación, dejando comprender lo que los estudiantes piensan respecto al aprendizaje de las Rotaciones.

- La entrevista semiestructurada (mixta), fue un diálogo que reconoció el origen de las frustraciones y dificultades que traían los estudiantes respecto a la comprensión del tema en estudio.

- La Observación aportó determinada información de comportamiento de los estudiantes, acciones, situaciones y casos en el aprendizaje, necesarios para acompañar a los estudiantes en el logro del aprendizaje de las Rotaciones.

- El modelo Van Hiele permitió comprender el pensamiento geométrico de los estudiantes en el logro de los niveles de Van Hiele, verificándose que la mayoría de los estudiantes, se ubicaron en el nivel 2, nivel de Análisis, por considerar que esto representa el cumplimiento con el objetivo de establecer los elementos necesarios para alcanzar la comprensión de la isometría de los ejercicios planteados, identificando y analizando partes y propiedades de las figuras rotadas, percibiendo componentes y propiedades de la rotación.

- El Módulo de Aprendizaje, sección Rotaciones impulsó el desarrollo del pensamiento geométrico durante la práctica de los ejercicios propuestos en tanto que la entrevista mixta y la observación brindaron información para atender los problemas de aprendizaje ambos instrumento y técnicas permitieron rendir una prueba formativa que ubicó a los estudiantes en su mayoría en el nivel 2 (análisis) con un buen camino hacia el logro del nivel 3 (clasificación), alcanzando el objetivo propuesto y el logro de aprendizaje.

- Las Guías de Instrucción Programada de característica constructivista componente del módulo de aprendizaje, concedió a los estudiantes del grupo experimental un progreso satisfactorio en los niveles 1 y 2 de Van Hiele permitiéndoseles a los estudiantes la construcción, paso a paso, de conceptos y desarrollo de ejercicios planteados.

- Las actividades con el software Geo Gebra comprometieron la realidad por ser dinámica, diversa y estar dirigida al significado de las acciones humanas. De esa manera, dieron importancia a la práctica social, a la comprensión y significación, lo que permitió tener una relación de participación comuni- 
cativa entre el docente y el estudiante, entorno a las acciones del paradigma interpretativo, permitiendo dar respuesta a la pregunta de investigación.

\section{RECOMENDACIONES}

- Una vez concluido el artículo, se considera adecuado aplicar la investigación cualitativa o mixta en investigaciones de educación por ser la educación un fenómeno complejo que tiene lugar en todos los ámbitos de la vida social en la cual intervienen diferentes disciplinas de las ciencias sociales.

- Analizar y comprobar con mayor detenimiento la búsqueda de la razón del porque los estudiantes no alcanzan los niveles completos de Van Hiele que le compete al nivel escolar, a fin de ayudarlos a madurar geométricamente para alcanzar el nivel inmediato superior.

- Trabajar en mejorar el modelo de Van Hiele, utilizando en este artículo para determinar actividades para alcanzar los niveles de Van Hiele en la Rotación.

- Extender los estudios expuestos en este trabajo de investigación al estudio de otros campos de la matemática, como son el álgebra, la aritmética y la estadística.

\section{REFERENCIAS BIBLIOGRÁFICAS}

Agencia de Calidad de la Educación (s/f). Evaluación formativa. Recuperado de http://www.agenciaeducacion.cl/evaluaciones/evaluacion-formativa/

Aravena, M. y Caamaño, C. (2013). Niveles de razonamiento geométrico en estudiantes de establecimiento municipalizados de la región del Maule, Talca, Chile. Revista Latinoamericana de Investigación en Matemática Educativa 16(2), 139-178. https://doi.org/10.12802/relime.13.1621

Blanco, L. (2015). Aportes de autores a la E/A de la Geometría. El Modelo de Van Hiele para la E/A de la Geometría. Recuperado de www.eweb.unex.es/ eweb/ljblanco/documentos/ModeloVanHiele.doc

Coxeter, H. y Greitzer, S. (1967). Geometry Revisited. Washington D.C.: The Mathematical Association of America (Inc.).

Fouz, F. (2005). Modelo de Van Hiele para la didáctica de la geometría. En Raúl Ibáñez y Marta Macho, Un paseo por la geometría 2004/2005 (pp. 67-81). Bilbao: Departamento de Matemáticas, Universidad del País Vasco. Recuperado de http://cimm.ucr.ac.cr/ciaem/articulos/universitario/materiales/Modelo\%20de\%20Van\%20Hiele\%20para\%20la $\% 20$ did\%C3\%A1ctica\%20de\%20la\%20Geometr\%C3\%ADa. ${ }^{*}$ Fouz, $\% 20$ 
Fernando\%3B\%20\%20De\%20Donosti,\%20Berritzegune. *Fernando\%20Fouz,\%20Berritzegune\%20de\%20Donosti.pdf

Goncalves, R. (2006). ¿Por qué los estudiantes no logran un nivel de razonamiento en la geometría? Revista Ciencias de la Educación, 6(1),83-98.

Gürtler, L. y Günter, H. (2007). Modos de pensar y estrategias de la investigación cualitativa. Liberabit. Revista Peruana de Psicología, 13, 37-52.

Gutiérrez, A. y Jaime, A. (1998). Geometría y algunos aspectos generales de la educación matemática. Bogotá, Colombia: Una empresa docente y Grupo Editorial Iberoamérica.

Gutiérrez, A., Jaime, A. y Fortuny, J. (1991). Alternative Paradigm to Evaluate the Acquisition of the van Hiele Levels. Journal for Research in Mathematics Education, 22(3), 237-251.

Heinz, K. y Schiefelbein, E. (2003). Instrucción Programada Capítulo XIII. En 20 Modelos Didácticos para América Latina (pp. 91-97). Washington, D.C.: Secretaría General de la Organización de los Estados Americanos (OEA).

Jaime, A. y Gutiérrez, A. (1990). Una propuesta de fundamentación para la enseñanza de la geometría: el modelo de van Hiele. En S. Linares y M.V. Sánchez (eds.), Teoría y práctica en educación matemática (pp. 295-384). Sevilla: Alfar. www.uv.es/-gutierre/archivos1/textospdf/JaiGut90.pdf

Jaime, A. y Gutiérrez, A. (1993). Aportaciones a la interpretación y aplicación del modelo de van Hiele: la enseñanza de las isometrías del plano. La evaluación del nivel de razonamiento (Tesis doctoral). Universidad de Valencia. https:// www.uv.es/gutierre/archivos1/textospdf/Jai93.pdf

Katayama, R. (2014). Introducción a la investigación cualitativa. Lima, Perú: Fondo Editorial de la Universidad Inca Garcilaso de la Vega.

Lobo, N. (2004). Aplicación del modelo propuesto en la Teoría de Van Hiele para la enseñanza de la geometría. Multiciencias, 4(1). Universidad de Zulia, Venezuela. http://www.redalyc.org/articulo.oa?id=90440104

Martínez-Salanova, E. (s/f). Concepción de la instrucción según J. Bruner. Recuperado de http://educomunicacion.es/didactica/32_instruccion_ bruner.htm

Rodríguez, G., Gil, J. y García, E. (1999). Metodología de la investigación cualitativa. Málaga, España: Ediciones Aljibe.

Universidad Internacional de Valencia (14 de febrero de 2015). Influencia de los factores sociales y familiares en el bajo rendimiento en el aprendizaje. Recuperado de: https://www.universidadviu.es/influencia-de-los-factoressociales-y-familiares-en-el-bajo-rendimiento-en-el-aprendizaje/ 
Vargas, G. y Gamboa, R. (2013). El modelo de Van Hiele y la enseńanza de la geometría. Uniciencia, 27(1), 74-94. Recuperado de http://www.revistas. una.ac.cr/index.php/uniciencia/article/view/4944

Wagner, E. (1993). Construçôes geométricas. Río de Janeiro, Brasil: SBM.

Yukavetsky, G. (2003). La elaboración de un módulo instruccional. Humacao, Puerto Rico: Centro de Competencias de la Comunicación. Universidad de Puerto Rico. http://academic.uprm.edu/ -marion/tecnofilia2011/ files/1277/CCC_LEDUMI.pdf 